\title{
The relationship between adolescent rumination and maternal rumination, criticism and positivity
}

Article

Published Version

Douglas, J. L., Williams, D. and Reynolds, S. (2017) The relationship between adolescent rumination and maternal rumination, criticism and positivity. Behavioural and Cognitive Psychotherapy, 45 (3). pp. 300-311. ISSN 1469-1833 doi: https://doi.org/10.1017/S1352465816000588 Available at https://centaur.reading.ac.uk/69948/

It is advisable to refer to the publisher's version if you intend to cite from the work. See Guidance on citing.

To link to this article DOI: http://dx.doi.org/10.1017/S1352465816000588

Publisher: Cambridge University Press

All outputs in CentAUR are protected by Intellectual Property Rights law, including copyright law. Copyright and IPR is retained by the creators or other copyright holders. Terms and conditions for use of this material are defined in the End User Agreement.

www.reading.ac.uk/centaur 
Central Archive at the University of Reading

Reading's research outputs online 


\title{
The Relationship between Adolescent Rumination and Maternal Rumination, Criticism and Positivity
}

\author{
Jessica L. Douglas ${ }^{1}$ \\ Children's Services, Addenbrooke's Hospital, Cambridge University Hospitals NHS Foundation Trust, \\ Cambridge, UK
}

Deirdre Williams

Honorary Lecturer, Department of Clinical Psychology, Norwich Medical School, University of East Anglia, Norwich, UK

Shirley Reynolds

Charlie Waller Institute, School of Psychology and Clinical Language Sciences, University of Reading, Reading, UK

\begin{abstract}
Background: Rumination predicts depression in adults and adolescents. The development of rumination has been linked to parenting practices, but only limited research has investigated this and research has tended to rely on self-report parenting measures. Aims: To investigate the relationship between female adolescent rumination and maternal modelling, criticism and positivity using an observational measure of parental behaviour. Method: A cross-sectional design was used. Daughters aged 16-18 years and their mothers $(n=154$ dyads) completed questionnaire measures of rumination and affect. Mothers of girls with rumination scores in the upper and lower quartile (both $n=26$ ) also completed the Five Minute Speech Sample, which was used to measure maternal criticism and positivity. Results: Mothers of low rumination girls made significantly more positive comments about their daughters than the mothers of high ruminators. Mothers made very few critical comments. Self-reported rumination was not correlated in mothers and daughters, suggesting a lack of support for the potential role of modelling. Conclusion: Overall, low maternal positivity was associated with rumination in female adolescents. There was no evidence that maternal rumination or criticism were associated with adolescent rumination. The results suggest a number of implications for future research, including the need for prospective longitudinal studies using observational parenting measures.
\end{abstract}

Keywords: Adolescents, rumination, brooding, parental behaviour, maternal, depression.

Correspondence to Jessica Douglas, Addenbrooke's Hospital, Cambridge, UK.

Email: jessica.douglas@ addenbrookes.nhs.uk

${ }^{1}$ Former Trainee Clinical Psychologist, Department of Clinical Psychology, Norwich Medical School, University of East Anglia, Norwich, UK. 


\section{Introduction}

According to Nolen-Hoeksema's (1987, 1991) Response Styles Theory, rumination exacerbates low mood. Rumination refers to a perseverative cognitive process and it has been defined as 'repetitively focusing on the fact that one is depressed; on one's symptoms of depression; and on the causes, meanings, and consequences of depressive symptoms' (NolenHoeksema, 1991, p. 569).

Rumination predicts depression in cross-sectional and longitudinal studies (for a review, see Nolen-Hoeksema et al., 2008). Women and girls are more likely to engage in rumination than males, and ruminative thinking appears to mediate the gender difference in depressive symptoms (e.g. Butler and Nolen-Hoeksema, 1994; Rood et al., 2009). Consequently, rumination may explain why females are twice as likely as men to experience depression (Nolen-Hoeksema, 1987).

Rumination has also been identified in numerous other psychological disorders (NolenHoeksema and Morrow, 1991; Kocovski et al., 2005; Guastella and Moulds, 2007). It has therefore been suggested that rumination is potentially an important transdiagnostic process (Harvey et al., 2004).

Understanding the developmental origins of rumination has been highlighted as a critical area of research (Nolen-Hoeksema et al., 2008). Nolen-Hoeksema (1991) hypothesized that parenting factors may play a role in the development of rumination. Firstly, she argued that children of depressed mothers tend to have difficulty regulating their own negative affect and she suggested that a tendency to ruminate may be transmitted via modelling (NolenHoeksema, 1991). She also suggested that critical and controlling parenting behaviours may mean children fail to develop active coping strategies which could lead them to use more passive styles of coping, such as rumination (Nolen-Hoeksema, 1991). Additionally, Gaté et al. (2013) have suggested an important role for parental positivity. They suggest that if active problem solving is not met with positive reinforcement from parents, such as praise, then these adaptive behaviours may be less likely to continue. Consequently, Gaté et al. (2013) suggest that low levels of parental positivity may lead to passive modes of responding, including rumination. However, to date, these hypotheses have received little research attention.

If mothers are modelling ruminative coping styles to their children and their children are copying them, then we would expect there to be a significant positive correlation between mother and child rumination scores. Whilst there could be alternative explanations for a positive association between these variables, a lack of correlation would be hard to explain if there is a modelling process occurring, as hypothesized by Nolen-Hoeksema (1991). Two studies found no correlation in children aged 8-12 years (Gardner and Epkins, 2012; Gibb et al., 2012). However, Stroud and Fitts (2015) found a positive association with mothers and their older children (mean 12.4 years). If a positive association can be explained by parental modelling, then the possible development of rumination via parental modelling may be developmentally sensitive and emerge as young people enter adolescence, a key risk period for depression (Rood et al., 2009).

Other parental behaviours have been shown to correlate with rumination. For example, parental control is correlated with adolescent rumination (e.g. Spasojevic and Alloy, 2002; Manfredi et al., 2011; Hilt et al., 2012; Vatanasin et al., 2012). Additionally, one study found that low maternal-adolescent relationship quality and high maternal encouragement of avoidance and denial responses to stressors was associated with adolescent rumination (Stroud 
and Fitts, 2015). However, all of these studies have used self-report measures of parenting which are subject to bias.

In a prospective longitudinal study of 163 adolescents and their mothers, Gaté et al. (2013) found that low levels of observed positive maternal behaviour predicted an increase in rumination scores over time for girls but not boys, and that this increase in rumination mediated the relationship between maternal positivity and depressive symptoms in daughters. Gaté et al. (2013) also explored the role of observed aggressive maternal behaviour, although there was no association with adolescent rumination. This was a broad measure and therefore this study was not able to isolate the specific impact of maternal critical comments on emerging adolescent rumination.

Given the results of Gaté et al. (2013) and the observed differences in the prevalence of depression and rumination in males and females (e.g. Twenge and Nolen-Hoeksema, 2002; Jose and Brown, 2008) it may be useful to explore the impact of parental behaviour towards male and female offspring separately. Additionally, research has revealed larger effect sizes for the relationship between same-sex parenting styles than opposite-sex parenting styles on depressive symptoms (Alloy et al., 2006). Consequently, focusing on mother-daughter relationships might have the strongest clinical implications.

The aim of the current study was to explore the relationship between rumination in adolescent females and maternal rumination, criticism and positivity. If mothers are transmitting ruminative responses to daughters via modelling then we would expect rumination in mothers and their daughters to be positively correlated. Similarly, if maternal criticism is a risk factor for rumination in daughters and if maternal positivity is protective, these behaviours observed directly from mothers should be significantly associated with adolescent rumination. Additionally, as mother and daughter affect are likely to be related to both daughter rumination and parenting style (Lovejoy et al., 2000; Nolen-Hoeksema et al., 2008; Rood et al., 2009), questionnaires ascertaining mother and daughter affect were used in order to see if any statistically significant findings remained after controlling for mother and daughter affect.

\section{Method}

\section{Participants}

Two hundred and two girls aged 16-18 years were recruited from high schools in the East and South East of England. Their mothers were also invited to take part. One hundred and fifty four dyads completed self-report questionnaire measures of rumination and affect. The majority of mothers (75.3\%) and daughters (77.6\%) were of white British ethnicity.

On the basis of the daughter's rumination score, an upper and lower quartile group were identified and their mothers were invited to complete the Five Minute Speech Sample (FMSS) by telephone. Thirty-one mothers of high ruminators and 30 mothers of low ruminators were eligible to participate, and 26 mothers in each group were recruited as indicated by power calculations (see Fig. 1 for recruitment flow chart).

\section{Measures}

Ruminative Responses Scale (Nolen-Hoeksema and Morrow, 1991). The Ruminative Responses Scale (RRS) is a 22-item self-report measure of rumination. It has good internal 


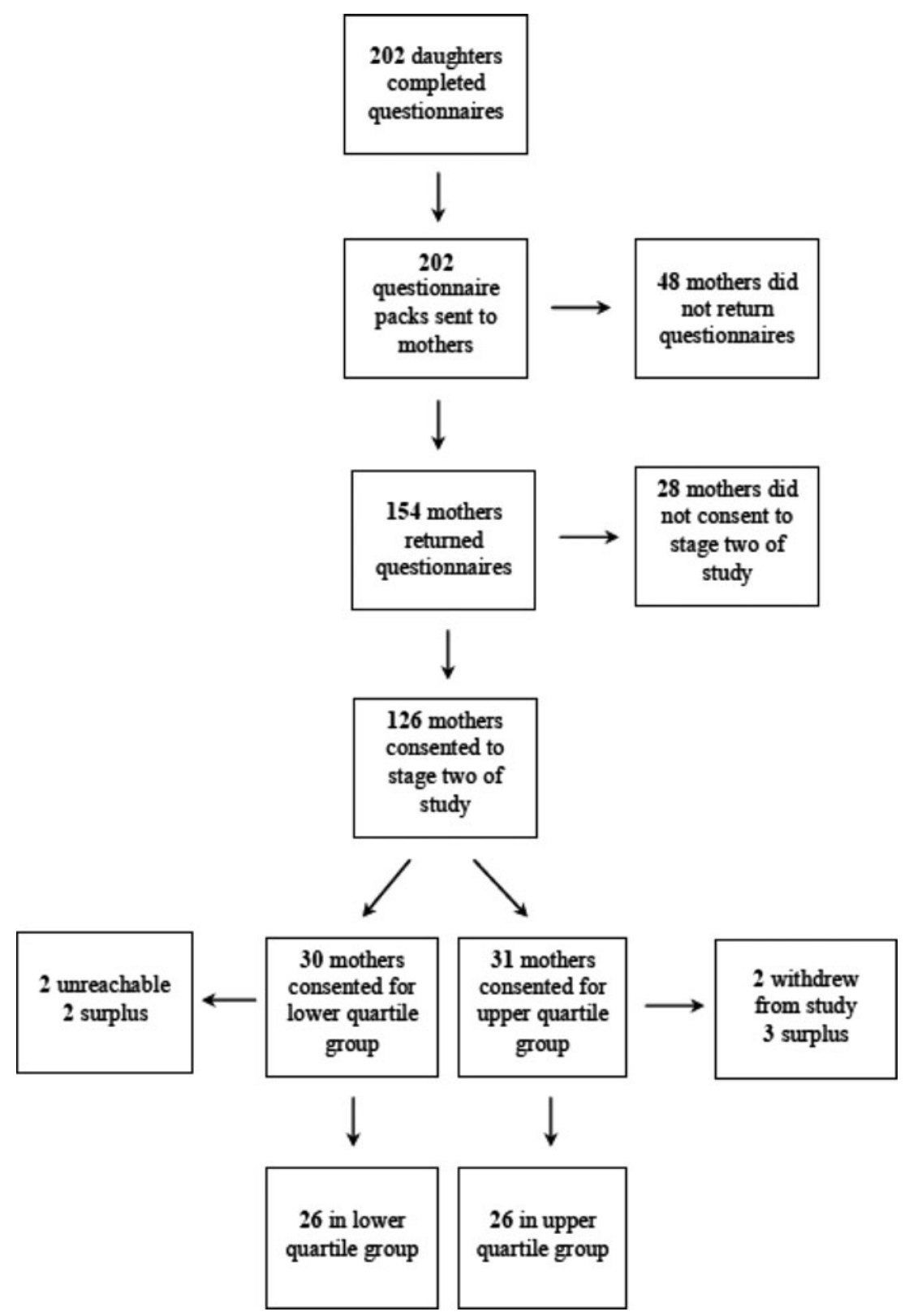

Figure 1. Flow of participants through stages 1 and 2 of the study

reliability, with Cronbach's alpha ( $\alpha$ ) values ranging from .88 to .93 in adult (Luminet, 2004) and adolescent samples (Kercher and Rapee, 2009). Scores on the RRS predict concurrent and prospective depressive symptoms in adults (e.g. Nolen-Hoeksema et al., 2008) and adolescents (e.g. Kercher and Rapee, 2009). 
In response to criticisms about the content validity of the RSS (i.e. that some items may overlap with depression; e.g. Conway et al., 2000), a two-factor scale from the RRS items has been developed: 'brooding' and 'reflective pondering' (Treynor et al., 2003). Reflective pondering is a form of contemplation which shows only a weak relationship with depressive symptoms and does not mediate the gender difference in depression (Treynor et al., 2003). The brooding subscale has good concurrent and predictive validity regarding its relationship with depressive symptoms and the subscale mediates the gender difference in depression (Treynor et al., 2003). The five-item brooding subscale has acceptable internal validity in nonclinical adult (Cronbach's $\alpha=.77$; Treynor et al., 2003) and adolescent samples (Cronbach's $\alpha=.79$; Cox et al., 2012). The brooding subscale was used to assess rumination in this study.

Positive and Negative Affect Scale (Watson et al., 1988). The Positive and Negative Affect Schedule (PANAS) consists of two 10-item subscales, measuring positive affect (PA) and negative affect (NA). Participants are asked to rate how they have been feeling over 'the last few weeks'. The PANAS has good internal reliability when used with adults (Crawford and Henry, 2004) and adolescents (Joiner et al., 1996; Melvin and Molloy, 2000; Molloy et al., 2001). It also has good convergent and divergent validity with alternative measures of anxiety and depression in both adult (Crawford and Henry, 2004) and youth samples (Joiner et al., 1996).

The Five Minute Speech Sample (Magana et al., 1986; Daley et al., 2003). The Five Minute Speech Sample (FMSS; Magana et al., 1986) originated within adult mental health to measure parental expressed emotion. Increasingly, the measure has been used in a wider range of contexts and research has demonstrated that a high frequency of critical comments and a low frequency of positive comments are related to child and adolescent psychopathology (McCarty and Weisz, 2002; Vostanis et al., 1994; Wambolt et al., 2000). Daley et al. (2003) adapted the coding system to be more developmentally appropriate for younger samples and some research has used individual subscales to assess specific components of parental behaviour. For example, one study utilizing the two frequency counts, critical and positive comments, found excellent inter-rater reliability when used with the mothers of non-clinical adolescents ( $r=.96$, for positive comments, $r=1.0$ for critical comments; Clark and Coker, 2009). These two subscales were also employed in the current study.

During the FMSS, the parent is asked to comment on their thoughts and feelings about their child for five minutes without any interruptions or comments from the researcher. The FMSS was collected over the phone. Beck et al. (2004) compared FMSS ratings administered by phone and face-to-face and found $100 \%$ agreement between ratings obtained via these two methods.

\section{Procedure}

The study was advertised to female students aged 16-18 years in four high schools via email, posters and through a short talk at lunchtime or during assembly. Participants completed questionnaires at school with the researcher present. They then took a questionnaire pack home to their mothers and completed packs were returned to the high school office. Mothers could indicate on their form whether or not they wished to participate in stage two of the study. This was a telephone call to administer the Five Minute Speech Sample. Seventy-six 
per cent of mothers (154/202) returned questionnaires and 126 consented to the telephone call (62\% of $n=202$; see Fig. 1). Analyses comparing mothers who did ( $n=126)$ or did not $(n=28)$ consent to complete the telephone call revealed that non-consenters were more likely to be single (Fisher's exact test: $p=.043$ ) and to be from a non-white British background $\left(\chi_{1}^{2}=8.71, p<.01\right)$, although there were no significant differences between these groups according to age (mean difference $=0.852,95 \% \mathrm{CI}-0.60$ to 2.46 ) or educational level (mean difference $=0.326,95 \% \mathrm{CI}-1.23$ to 1.96 , all group comparisons two-tailed).

Consenting mothers of daughters scoring in the upper or lower quartile of the brooding subscale were selected. The total brooding scores of daughters in the lower quartile ranged from 6 to 9 and the total scores ranged from 14 to 20 in the upper quartile. Mothers of high and low ruminating daughters (top and bottom quartile) were invited to take part in the FMSS. Two participants from the low rumination group could not be contacted and two participants from the high rumination group no longer wanted to participate when contacted. This left 28 potential participants in the low rumination group and 29 in the high rumination group. Each participant was contacted in turn until a required sample size of 26 was reached for each group.

During the collection of the FMSS, mothers were asked to go into a room on their own where there would not be any distractions. The speech samples were recorded and transcribed and then rated for the frequency of critical and positive comments by the first author. Fifteen per cent of FMSSs were also blindly rated by an experienced FMSS coder. There was high agreement between the two sets of ratings (intraclass correlations $\geq .98$ ).

\section{Statistical approach}

The distribution of the data was assessed visually using histograms, stem and leaf diagrams and Q-Q plots. Normality was explored by examining the significance of the skewness and kurtosis values and also the significance of the Shapiro-Wilk test. All tests employed considered significance at the $p=.05$ level. Data were transformed as appropriate. If the data did not display a normal distribution following transformation, then bootstrapping was used with the original untransformed data. Bootstrapping estimates the characteristics of the sampling distribution by using the sample data itself. It does not change the statistic in question (e.g. the mean difference) but it estimates how accurate it is (Wright et al., 2011). Statistical analyses were conducted using SPSS version 19, which supports bootstrapping procedures for a number of different tests. The default setting of 1000 bootstraps was used and bias corrected and accelerated confidence intervals were selected, as recommended by Field (2013).

\section{Results}

Descriptive data for brooding and affect scores for the 154 mother-daughter dyads and for the high and low rumination groups $(n=26$ each) are shown in Table 1. For participants in this sample, the internal reliability of the brooding subscale (mothers' Cronbach's $\alpha=.73$, daughters' Cronbach's $\alpha=.69$ ) and the PANAS was acceptable (mothers' Cronbach's $\alpha$ PA: .86 , NA: .84; daughters' Cronbach's $\alpha$ PA: .81, NA: .72).

For daughters there was a significant, positive correlation between rumination and negative affect ( $r=.39,95 \%$ CI .231 to .532 , two-tailed) but no relationship between rumination and 
Table 1. Mother and daughter brooding and affect scores

\begin{tabular}{|c|c|c|c|c|c|c|c|}
\hline \multirow[b]{2}{*}{ Measure (total score) } & \multirow[b]{2}{*}{ Participant } & \multicolumn{2}{|c|}{$\begin{array}{l}\text { Whole group } \\
\quad(n=154)\end{array}$} & \multicolumn{2}{|c|}{$\begin{array}{l}\text { Low rumination } \\
\text { group }(n=26)\end{array}$} & \multicolumn{2}{|c|}{$\begin{array}{c}\text { High } \\
\text { rumination } \\
\text { group }(n=26)\end{array}$} \\
\hline & & Mean & SD & Mean & SD & Mean & SD \\
\hline \multirow[t]{2}{*}{ RRS brooding } & Mother & 9.31 & 2.84 & 9.38 & 2.93 & 10.00 & 2.56 \\
\hline & Daughter & 11.34 & 3.25 & 7.62 & .94 & 15.65 & 1.62 \\
\hline \multirow[t]{2}{*}{ PANAS positive affect } & Mother & 35.51 & 6.50 & 37.60 & 5.36 & 34.50 & 6.15 \\
\hline & Daughter & 31.26 & 6.02 & 31.81 & 5.11 & 30.56 & 6.62 \\
\hline \multirow[t]{2}{*}{ PANAS negative affect } & Mother & 18.85 & 6.24 & 17.77 & 5.44 & 19.57 & 6.32 \\
\hline & Daughter & 21.01 & 5.15 & 19.00 & 4.99 & 23.27 & 6.17 \\
\hline
\end{tabular}

RRS, ruminative responses scale; PANAS, positive and negative affect scale.

positive affect ( $r=-.08,95 \% \mathrm{CI}-.232$ to .084$)$. Daughter rumination was not correlated with mother negative affect ( $r=.07,95 \% \mathrm{CI}-.087$ to .227 ) or mother positive affect $(r=-.11$, $95 \% \mathrm{CI}-.271$ to .038 ). There was also no correlation between mother and daughter rumination ( $r=.05,95 \% \mathrm{CI}-.115$ to .200 , two-tailed). This relationship remained non-significant when controlling for daughter negative affect ( $b=-.02,95 \% \mathrm{CI}-.19$ to $.15, p=.86$, two-tailed).

Affect scores and maternal comments were examined within the sub-groups of low and high ruminator girls. For daughters there was a significant difference in negative affect between the high and low ruminator groups $\left(t_{50}=2.78, p=0.008\right.$, two-tailed) but no significant difference in their positive affect $\left(t_{50}=.76, p=0.449\right.$, two-tailed; see Table 1$)$. There was also no between-groups difference in negative affect for mothers $\left(t_{50}=1.12, p=.270\right.$, two-tailed $)$. There was a trend for mothers of low rumination daughters to report higher positive affect than the mothers of high rumination daughters $\left(t_{50}=1.94, p=0.059\right.$, two-tailed).

Maternal positivity and criticism were assessed by observation using the FMSS. Mean positive comments were $6.08(\mathrm{SD}=3.15,95 \% \mathrm{CI} 4.76$ to 7.32$)$ for mothers of low rumination daughters and 3.38 ( $\mathrm{SD}=2.48,95 \% \mathrm{CI} 2.58$ to 4.28 ) for mothers of high rumination daughters. A bootstrapped independent-samples $t$-test indicated that this was statistically significant ( $p=.002,95 \%$ CI .78 to 4.16 , two-tailed). Cohen's $d$ revealed a large effect size $(d=.95)$. To control for the potential impact of affect on maternal behaviour, maternal positive affect and daughter negative affect were controlled. This calculation was completed in R as bootstrapping is not well supported for ANOVA in SPSS version 19. There was still a significant group difference in positive comments when controlling for these two affect variables $(\beta=2.95, \mathrm{SE}=.90,95 \% \mathrm{CI}-4.41$ to -.84 , two-tailed $)$.

The frequency of critical comments in this sample was extremely low. One mother in the low rumination group made three critical comments about her daughter and the other 25 mothers in the group made no critical comments. In the high rumination group, five mothers made critical comments about their daughters, with three mothers making one critical comment and two mothers making two critical comments. Due to the low frequency of critical comments, each observation was dichotomized as either containing no critical comments or one or more critical comments. Fisher's exact test revealed a non-significant group difference $(p=0.19$, two-tailed). 


\section{Discussion}

This study investigated the relationship between adolescent rumination and maternal rumination, criticism and positivity using an observational measure of parental behaviour, the FMSS. Overall, the results showed that the mothers of low ruminating daughters were significantly more positive about their daughters and that this effect remained when controlling for mother and daughter affect variables. However, the relationship between maternal criticism and daughter rumination was difficult to explore due to a low frequency of critical comments in this sample. Rumination was not correlated in mothers and daughters, suggesting a lack of support for Nolen-Hoeksema's (1991) modelling hypothesis.

Adolescent girls are at particularly high risk of developing depression (Rood et al., 2009). Depression during adolescence is difficult to treat and also increases the risk of lifelong mental distress and physical health problems (Bardone et al., 1998; Birmaher et al., 1998). It is therefore important to identify internal and environmental variables that may mediate this risk so that effective prevention and treatment programmes can be developed. These results suggest that rumination in adolescent girls, which itself is a predictor of depression onset (Nolen-Hoeksema et al., 2008), is associated with the absence of positive maternal behaviours. These findings are consistent with Gaté et al. (2013) who found that low maternal positivity predicted an increase in rumination in daughters between 12 and 15 years. Interestingly, they did not find a significant cross-sectional relationship between maternal positivity and rumination in females at any time point. They suggest that the impact of parenting on rumination may emerge over time and this may explain why a significant cross-sectional result was found in the present study with older girls (aged 16-18 years).

Gaté et al. (2013) argue that low maternal positivity may mean that adaptive behaviours in their children, which could serve as alternatives to rumination, are not reinforced and therefore are less likely to continue. However, exactly why low maternal positivity is related to rumination in their children requires further investigation. It is also possible that low maternal positivity means that children develop low self-efficacy, which could leave them vulnerable to rumination. These mechanisms require further exploration.

Nolen-Hoeksema (1991) hypothesized that a tendency to ruminate may be transmitted via modelling. In the present study, there was no relationship between mother and daughter rumination, in line with the findings of Gibb et al. (2012) and Gardner and Epkins (2012) and in contrast to those of Stroud and Fitts (2015). Whilst a positive correlation between mother and daughter rumination could be explained by processes other than modelling, the finding of no correlation in this study is difficult to explain if rumination is at least partly transmitted via modelling. However, it could be that a different socialization process is occurring. Rose (2002) suggested that co-rumination may be an important factor in the development of emotional difficulties. Co-rumination is an interpersonal process which can be defined as 'discussing the same problem repeatedly, mutual encouragement of discussing problems, speculating about problems, and focusing on negative feelings' (Rose, 2002, p. 1830). Exploring whether parental co-rumination is correlated with offspring rumination either cross-sectionally or prospectively could form a useful avenue for further investigation. Additionally, future research could investigate if parental modelling of positive coping skills is protective against the development of rumination during adolescence.

Nolen-Hoeksema (1991) also suggested that parental criticism may lead to rumination in offspring. The relationship between parental criticism and offspring rumination has not 
been explored previously. However, Gaté et al. (2013) did investigate the role of aggressive parenting behaviours in the development of rumination and found no association. As with critical comments in the present study, Gaté et al. (2013) also found a very low frequency of maternal aggressive behaviours in their sample. This may reflect the fact that both studies used non-clinical samples. This could be seen as a weakness of both studies. However, it may be more appropriate to use non-clinical samples when investigating the developmental origins of a psychological phenomenon. It is possible that a focus on the relationship between rumination and low positive interactions between mother and daughters, rather than a focus on negative parental behaviours, may be a more useful focus for future research and eventually in the development of prevention or treatment programmes.

The findings of this study add to the extant existing literature on the relationship between parental behaviours and rumination in their children. Other strengths of this study include the fact that it is one of the only studies to use an observational measure of parental behaviour, although this measure did not observe interactions between mothers and daughters directly. A significant limitation is that this study was correlational in nature and so it is not possible to infer causality. Therefore, it cannot be concluded that low maternal positivity causes the development of rumination in adolescent females. It could be that daughters who tend to ruminate elicit less praise from their parents. Additionally, analyses showed that of the mothers that completed the questionnaires, those that were single or from non-White British backgrounds were less likely to consent to the telephone call. Consequently, the results may not be as applicable to more socially and ethnically diverse samples.

Future research should use observational measures of parenting using prospective designs. Additionally, the role of fathers, siblings and peers could also be explored. Peer relationships may be especially influential as they become increasingly important at the stage in development when rumination is emerging (Furman and Buhrmester, 1992).

In summary, the results of this investigation indicate an association between low maternal positivity and higher rumination scores in adolescent daughters, even when controlling for mother and daughter affect. A lack of positive association between mother and daughter rumination undermines Nolen-Hoeksema's (1991) modelling hypothesis, although it may be worth investigating the role of co-rumination. Finally, the relationship between maternal criticism and daughter rumination could not be explored due to a low frequency of critical comments in this sample, similar to the findings of Gaté et al. (2013). Future research should aim to use observational measures of parenting, prospective designs and explore the role of other significant relationships.

\section{Acknowledgements}

We would like to thanks the teachers, mothers and daughters that made this project possible.

Financial support: This work was supported by a small internal funding source at the academic institution. No external funding was applied for.

Conflicts of interest: The authors have no conflicts of interest with respect to this publication.

Ethical standards: The authors assert that all procedures contributing to this work comply with the ethical standards of the relevant national and institutional committees on human experimentation and with the Helsinki Declaration of 1975, and its most recent revision. 
Ethical approval was granted by the Research Ethics Committee at the University where the research was based.

\section{References}

Alloy, L., Abramson, L., Smith, J., Gibb, B. and Neeren, A. (2006). Role of parenting and maltreatment histories in unipolar and bipolar mood disorders: mediation by vulnerability to depression. Clinical Child and Family Psychology Review, 9, 23-64. doi: 10.1007/s10567-006-0002-4

Bardone, A., Moffitt, T., Caspi, A., Dickson, N., Stanton, W. and Silva, P. (1998). Adult physical health outcomes of adolescent girls with conduct disorder, depression, and anxiety. Journal of the American Academy of Child and Adolescent Psychiatry, 37, 594-601. doi: 10.1097/00004583-199806000-00009

Beck, A., Daley, D., Hastings, R. and Stevenson, J. (2004). Mothers' expressed emotion towards children with and without intellectual disabilities. Journal of Intellectual Disability Research, 48, 628-638. doi: 10.1111/j.1365-2788.2003.00564.x

Birmaher, B., Brent, A. and Benson, S. (1998). Summary of the practice parameters for the assessment and treatment of children and adolescents with depressive disorders. Journal of the American Academy of Child and Adolescent Psychiatry, 37, 1234-1238. doi: 10.1097/00004583-199811000-00029

Butler, L. and Nolen-Hoeksema, S. (1994). Gender differences in responses to depressed mood in a college sample. Sex Roles, 30, 331-346. doi: 10.1007/BF01420597

Clark, S. and Coker, S. (2009). Perfectionism, self-criticism and maternal criticism: a study of mothers and their children. Personality and Individual Differences, 47, 321-325. doi: 10.1016/j.paid.2009.03.020

Conway, M., Csank, P., Holm, S. and Blake, C. (2000). On assessing individual differences in rumination on sadness. Journal of Personality Assessment, 75, 404-425. doi: 10.1207/S15327752JPA7503_04

Cox, S., Funasaki, K., Smith, L. and Mezulis, A. (2012). A prospective study of brooding and reflection as moderators of the relationship between stress and depressive symptoms in adolescence. Cognitive Therapy and Research, 36, 290-299. doi: 10.1007/s10608-011-9373-z

Crawford, J. and Henry, J. (2004). The positive and negative affect schedule (PANAS): construct validity, measurement properties and normative data in a large non-clinical sample. British Journal of Clinical Psychology, 43, 245-265. doi: 10.1348/0144665031752934

Daley, D., Sonuga-Barke, E. and Thompson, M. (2003). Assessing expressed emotion in mothers of preschool ADHD children: psychometric properties of a modified speech sample. British Journal of Clinical Psychology, 42, 53-67. doi: 10.1348/014466503762842011

Field, A. (2013). Discovering Statistics Using IBM SPSS Statistics. London: Sage Publications.

Furman, W. and Buhrmester, D. (1992). Age and sex differences in perceptions of networks of personal relationships. Child Development, 63, 103-115. doi: 10.1111/j.1467-8624.1992.tb03599.x

Gardner, C. and Epkins, C. (2012). Girls' rumination and anxiety sensitivity: are they related after controlling for girl, maternal and parenting factors? Child Youth Care Forum, 41, 561-578. doi: 10.1007/s10566-012-9188-4

Gaté, M., Watkins, E., Simmons, J., Byrne, M., Schwartz, O., Whittle, S. et al. (2013). Maternal parenting behaviors and adolescent depression: the mediating role of rumination. Journal of Clinical Child and Adolescent Psychology, 42, 348-357. doi: 10.1080/15374416.2012.755927

Gibb, B., Grassia, M., Stone, L., Uhrlass, D. and McGeary, J. (2012). Brooding rumination and risk for depressive disorders in children of depressed mothers. Journal of Abnormal Child Psychology, 40, 317-326. doi: 10.1007/s10802-011-9554-y 
Guastella, A. and Moulds, M. (2007). The impact of rumination on sleep quality following a stressful life event. Personality and Individual Differences, 42, 1151-1162. doi: 10.1016/j.paid.2006.04.028

Harvey, A., Watkins, E., Mansell, W. and Shafran, R. (2004). Cognitive Behavioural Processes across Psychological Disorders: a Transdiagnostic Approach to Research and Treatment. Oxford: Oxford University Press.

Hilt, L., Armstrong, J. and Essex, M. (2012). Early family context and development of adolescent ruminative style: moderation by temperament. Cognition and Emotion, 26, 916-926. doi: 10.1080/02699931.2011.621932

Joiner, T., Catanzaro, S. and Laurent, J. (1996). Tripartite structure of positive and negative affect, depression, and anxiety in child and adolescent psychiatric inpatients. Journal of Abnormal Psychology, 105, 401-409. doi: 10.1037/0021-843X.105.3.401

Jose, P. and Brown, I. (2008). When does the gender difference in rumination begin? Gender and age differences in the use of rumination by adolescents. Journal of Youth and Adolescence, 37, 180-192. doi: 10.1007/s10964-006-9166-y

Kercher, A. and Rapee, R. (2009). A test of a cognitive diathesis-stress generation pathway in early adolescent depression. Journal of Abnormal Child Psychology, 37, 845-855. doi: 10.1007/s10802-009-9315-3

Kocovski, N., Endler, N., Rector, N. and Flett, G. (2005). Ruminative coping and postevent processing in social anxiety. Behaviour Research and Therapy, 43, 971-984. doi: 10.1016/j.brat.2004.06.015

Lovejoy, M., Graczyk, P., O'Hare, E. and Neuman, G. (2000). Maternal depression and parenting behavior: a meta-analytic review. Clinical Psychology Review, 20, 561-592. doi:10.1016/S0272-7358(98)00100-7

Luminet, O. (2004). Measurement of depressive rumination and associated constructs. In C. Papageorgiou and A. Wells (eds), Depressive Rumination: Nature, Theory and Treatment. Chichester: Wiley and Sons.

Magana, A., Goldstien, M., Karno, M., Miklowitz, D., Jenkins, J. and Falloon, I. (1986). A brief method for assessing expressed emotion in relatives of psychiatric patients. Psychiatry Research, 17, 203-212. doi: 10.1016/0165-1781(86)90049-1

Manfredi, C., Caselli, G., Rovetto, F., Rebecchi, D., Ruggiero, G., Sassaroli, S. and Spada, M. (2011). Temperament and parental styles as predictors of ruminative brooding and worry. Personality and Individual Differences, 50, 186-191. doi:10.1016/j.paid.2010.09.023

McCarty, C. and Weisz, J. (2002). Correlates of expressed emotion in mothers of clinically referred youth: an examination of the five-minute speech sample. Journal of Child Psychology and Psychiatry, 43, 759-768. doi: 10.1111/1469-7610.00090

Melvin, G. and Molloy, G. (2000). Some psychometric properties of the Positive and Negative Affect Schedule among Australian youth. Psychological Reports, 86, 1209-1212. doi: 10.2466/pr0.2000.86.3c.1209

Molloy, G., Pallant, J. and Cantas, A. (2001). A psychometric comparison of the positive and negative affect schedule across age and sex. Psychological Reports, 88, 861-862. doi: 10.2466/PR0.88.3.861-862

Nolen-Hoeksema, S. (1987). Sex differences in unipolar depression: evidence and theory. Psychological Bulletin, 101, 259-282. doi: 10.1037/0033-2909.101.2.259

Nolen-Hoeksema, S. (1991). Responses to depression and their effects on the duration of depressive episodes. Journal of Abnormal Psychology, 100, 569-582. doi: 10.1037/0021-843X.100.4.569

Nolen-Hoeksema, S. and Morrow, J. (1991). A prospective study of depression and posttraumatic stress symptoms after a natural disaster: the 1989 Loma Piesta earthquake. Journal of Personality and Social Psychology, 61, 115-121. doi: 10.1037/0022-3514.61.1.115

Nolen-Hoeksema, S., Wisco, B. and Lyubomirsky, S. (2008). Rethinking rumination. Perspectives on Psychological Science, 3, 400-424. doi: 10.1111/j.1745-6924.2008.00088.x 
Rood, L., Roelofs, J., Bogels, S., Nolen-Hoeksema, S. and Schouten, E. (2009). The influence of emotion-focused rumination and distraction on depressive symptoms in non-clinical youth: a metaanalytic review. Clinical Psychology Review, 29, 607-616. doi:10.1016/j.cpr.2009.07.001

Rose, A. (2002). Co-rumination in the friendships of girls and boys. Child Development, 73, 1830-1843. doi: $10.1111 / 1467-8624.00509$

Spasojevic, J. and Alloy, L. (2002). Who becomes a depressive ruminator? Developmental antecedents of ruminative response style. Journal of Cognitive Psychotherapy: An International Quarterly, 16, 405-419. doi: 10.1891/jcop.16.4.405.52529

Stroud, C. B. and Fitts, J. (2015). Rumination in early adolescent girls: interactive contributions of mother-adolescent relationship quality and maternal coping suggestions. Journal of Clinical Child and Adolescent Psychology, Epub ahead of print, 1-12. doi: 10.1080/15374416.2015.1094737

Treynor, W., Gonzalez, R. and Nolen-Hoeksema, S. (2003). Rumination reconsidered: a psychometric analysis. Cognitive Therapy and Research, 27, 247-259. doi: 10.1023/A:1023910315561

Twenge, J. and Nolen-Hoeksema, S. (2002). Age, gender, race, socioeconomic status and birth cohort difference on the children's depression inventory: a meta-analysis. Journal of Abnormal Psychology, 111, 578-588. doi: 10.1037/0021-843X.111.4.578

Vatanasin, D., Thapinta, D., Thompson, E. and Thunjaroenkul, P. (2012). Testing a model of depression among Thai adolescents. Journal of Child and Adolescent Psychiatric Nursing, 25, 195206. doi: 10.1111/jcap. 12012

Vostanis, P., Nicholls, J. and Harrington, R. (1994). Maternal expressed emotion in conduct and emotional disorders of childhood. Journal of Child Psychology and Psychiatry, 35, 365-376. doi: 10.1111/j.1469-7610.1994.tb01168.x

Wambolt, F., O'Connor, S., Wambolt, M., Gavin, L. and Klinnert, M. (2000). The five minute speech sample in children with asthma: deconstructing the construct of expressed emotion. Journal of Child Psychology and Psychiatry, 41, 887-898. doi: 10.1111/1469-7610.00676

Watson, D., Clark, L. and Tellegen, A. (1988). Development and validation of brief measures of positive and negative affect: the PANAS scales. Journal of Personality and Social Psychology, 47, 1063-1070. doi: 10.1037/0022-3514.54.6.1063.

Wright, D., London, K. and Field, A. (2011). Using bootstrap estimation and the plug-in principle for clinical psychology data. Journal of Experimental Psychopathology, 2, 252-270. doi: 10.5127/jep.013611. 